\title{
An unusual case of migration of Filshie clip
}

\section{Alka Verma, Odiri E Oteri}

\section{Case report}

A 53-year-old woman presented with a 4-week history of constant pain and a lump in the right groin. She also gave a history of low-grade fever, loss of weight and general malaise. There was no history of vaginal discharge or urinary symptoms. She was a housewife and had been in the same relationship for 19 years. The only relevant past history was that she had undergone laparoscopic sterilisation with Filshie clips 13 years previously. There was no history of recent surgery.

On admission to hospital she had a low-grade pyrexia. An exquisitely tender $5 \mathrm{~cm}$ diameter indurated mass was present in the right groin. Vaginal examination revealed marked right adnexal tenderness. The clinical impression was that of a lymph node mass or a psoas abscess. She was admitted and started on antibiotics. Investigations revealed a moderate neutrophil leucocytosis. Abdominal X-ray showed a sterilisation clip projected over the medial aspect of the right femoral head. The left clip appeared to be normally located. This was confirmed on a computed tomography (CT) scan, which revealed a right iliac fossa inflammatory lesion, possibly a tubal abscess, intimately related to the right tubal clip. There was no abdominal or pelvic lymphadenopathy.

Her pyrexia persisted and in view of the CT findings consent was obtained for exploratory laparotomy. This showed a right-sided extraperitoneal abscess containing $400 \mathrm{ml}$ pus. A Filshie clip was seen in the abscess cavity. There was no pus in the abdominal cavity. Both Fallopian tubes were adherent to the anterior abdominal wall. There was a $5 \mathrm{~cm}$ unilocular right ovarian cyst, which was also stuck to the anterior abdominal wall. The left ovary was normal. A Filshie clip was seen appropriately placed on the left Fallopian tube. The abscess was drained and a right ovarian cystectomy was performed. The patient made an uncomplicated postoperative recovery. Histology showed a benign ovarian serous cystadenoma. There was a thickened area at the base of the cyst, which contained multiple small abscesses. Special stains for bacteria, yeasts, fungi and tubercle bacilli were negative.

J Fam Plann Reprod Health Care 2007; 33(3): 212

(Accepted 12 October 2006)

Department of Obstetrics and Gynaecology, Division of Women and Children, Lincoln County Hospital, Lincoln, UK Odiri E Oteri, MBBS, MRCOG, Consultant Obstetrician and Gynaecologist

Alka Verma, MBBS, MRCOG, Specialist Registrar

Correspondence to: Dr A Verma, Department of Obstetrics and Gynaecology, Leicester Royal Infirmary, Leicester LE1 5WW, UK. E-mail: vineetalka@aol.com

\section{Discussion}

Laparoscopic Fallopian tube occlusion using Filshie clips is a common and popular method of contraception. This is mainly because of its safety, short recovery time and failure rate of only $0.2-0.5 \%$. Migration of Filshie clips following normal application is common, with an estimated incidence of over $20 \% .^{1}$ The pathophysiology is thought to be related to the speed of peritonealisation over the clip. After clip application, avascular necrosis occurs at the site, eventually leading to division of the tube. If peritonealisation is rapid the clip remains in situ; if it is slow, the clip falls off after transection of the tube and may migrate. ${ }^{1}$

Most clips that detach and migrate settle in the omentum, pouch of Douglas or paracolic gutters and rarely cause problems. ${ }^{1}$ They occasionally migrate to other sites. Migration followed by expulsion of Filshie clips from the body occurs very rarely and even less frequent is migration followed by abscess formation. In a prospective study involving over 6000 patients, which was presented to a United States Food and Drug Administration panel hearing in 1996, three clips were noted to have been expelled from the body via the vagina, urethra and rectum but in no case was any significant morbidity observed. Since then various other sites from which migrated Filshie clips have been retrieved have included anal fistula, ${ }^{2}$ urinary bladder ${ }^{3}$ and abdominal wall. ${ }^{4}$ One case has been described where both Filshie clips were extruded through the rectum..$^{5}$ The likely sequence of events in all these cases is a low-grade foreign body inflammatory response induced by the displaced clip. Localised inflammation and adhesion formation could lead to incorporation of the clip in the wall of a hollow viscus with eventual burrowing through the wall into the cavity and then expulsion via an orifice. The migration events occurred up to 7 years after application. The present case is unusual because the patient presented 13 years after the procedure. The migrated Filshie clip was associated with an abscess that extended anteriorly and formed a mass in the right groin and possibly also involved the ovarian cyst, which was stuck to the abdominal wall. It is also unusual in that the abscess did not rupture into the peritoneal cavity. The ovarian cyst itself was probably an incidental finding.

\section{Statements on funding and competing interests}

Funding None identified.

Competing interests None identified.

\section{References}

1 Filshie GM. Sterilisation. The Obstetrician and Gynaecologist 1999; 1: 29-31.

2 Hasan A, Evgenikos N, Daniel T, Gatongi D. Filshie clip migration with recurrent perianal sepsis and low fistula in ano formation. Br J Obstet Gynaecol 2005; 112: 1581

3 Miliauskas JR. Migration of a Filshie clip into the urinary bladder with abscess formation. Pathology 2003; 35: 356-357.

4 Krishnamoorthy M, Nysenbaum AM. Spontaneous extrusion of a migrating Filshie clip through the anterior abdominal wall. $J$ Obstet Gynaecol 2004; 24: 328-329.

5 Pandit M. Early extrusion of bilateral Filshie clips after laparoscopic sterilisation. Br J Obstet Gynaecol 2005; 112: 680.

\section{Visit the Faculty Website at www.ffprhc.org.uk}

